\title{
Divide or die
}

Cell proliferation and cell death might seem like opposing functions, but oncogenes such as E1A and MYC are able to initiate both. Apoptosis is thought to be a safety mechanism, and is induced when the oncogenic signal to proliferate is recognized as excessive, and hence likely to cause tumorigenesis. The mechanisms by which oncogenes induce cell death have not been clearly established. Zaher Nahle, Scott Lowe and colleagues have investigated this phenomenon using E1A, and have found that the E2F transcription factor, which promotes replication and hence proliferation, has a key role in coordinating these processes.

The authors first investigated whether overexpression of $E 1 A$ in mouse embryonic fibroblasts (MEFs) and normal diploid human fibroblasts (IMR90 cells) affected the protein levels of caspases - the effectors of cell death - and found that both initiator and effector caspases were upregulated by $5-15$-fold. A similar increase was observed in cells deficient for either ARF or p53, so E1A must upregulate caspases through a p53-independent pathway.

One of the key targets of E1A is retinoblastoma (RB), and $\mathrm{E} 1 \mathrm{~A}$ mutants that are unable to inactivate $\mathrm{RB}$ do not upregulate caspases. Similarly, $R B^{-/-}$MEFs expressed higher levels of caspases than wildtype cells. Introduction of wild-type $R B$, but not a tumour-derived mutant that can not bind $\mathrm{E} 2 \mathrm{~F}$, into RB-deficient cells represses this caspase expression, and implicates the E2F family of proteins in this apoptotic pathway. In fact, expression of E2F1 is sufficient to induce this caspase induction.

So, are caspases transcriptional targets of E2F1, or is the induction indirect? Northern blots revealed that caspase mRNA was increased by 5-15-fold (similar to the protein levels) when either E1A or E2F1 was expressed in IMR90 cells. Caspase mRNA levels also increase as cells enter $\mathrm{S}$ phase, which corresponds with the activity of E2F1 and the levels of cyclin A mRNA - a known E2F1 target. Analysis of caspase promoters provided further support that E2F could transcriptionally activate caspases, as several contain E2F1binding sites, and chromatin immunoprecipitation experiments confirmed this - $\mathrm{E} 2 \mathrm{~F} 1$ precipitates from E1A-expressing cells that contained sequences from the caspase-7 promoter. The caspase- 7 promoter was also able to drive transcription of the luciferase reporter gene expression increased by almost 18-fold - when E2F1 was expressed.

But what is the physiological role of this caspase induction? It is not sufficient to induce apoptosis and, instead, seems to sensitize cells to apoptotic stimuli, such as serum withdrawal and adriamycin treatment. E2F1 is also known to activate cytochrome $c$ release - a downstream event in the apoptotic pathway - via a p53-dependent pathway. The requirement for p53 in inducing apoptosis could be recapitulated, at least in part, by either introducing BAX - a pro-apoptotic protein that facilitates cytochrome $c$ release - to $\mathrm{TP}^{-/-} \mathrm{BAX^{-- }}$ cells expressing E2F, or by directly microinjecting cytochrome $c$ into $T P 53^{-/-} R B^{-/-}$cells. Under these conditions, caspase induction by E2F1 is able to enhance apoptosis, underscoring the cooperation of the $\mathrm{p} 53$ dependent and -independent pathways in inducing apoptosis.

So, the E1A oncogene coordinates division and death by using the same machinery - E2F - to initiate both processes. Whether other oncogenes operate in the same way remains to be determined.

Emma Greenwood

64) References and links ORIGINAL RESEARCH PAPER Nahle, Z. et al. Direct coupling of the cell cycle and cell death machinery by E2F. Nature Cell Biol. 4, 859-864 machin

FURTHER READING Trimarchi, J. M. \& Lees, J. A. Sibling rivalry in the E2F family. Nature Rev. Mol. Cell Biol. 3, 11-20 (2002)

WEB SITE

Scott Lowe's lab:

http://www.cshl.org/public/SCIENCE/lowe.html

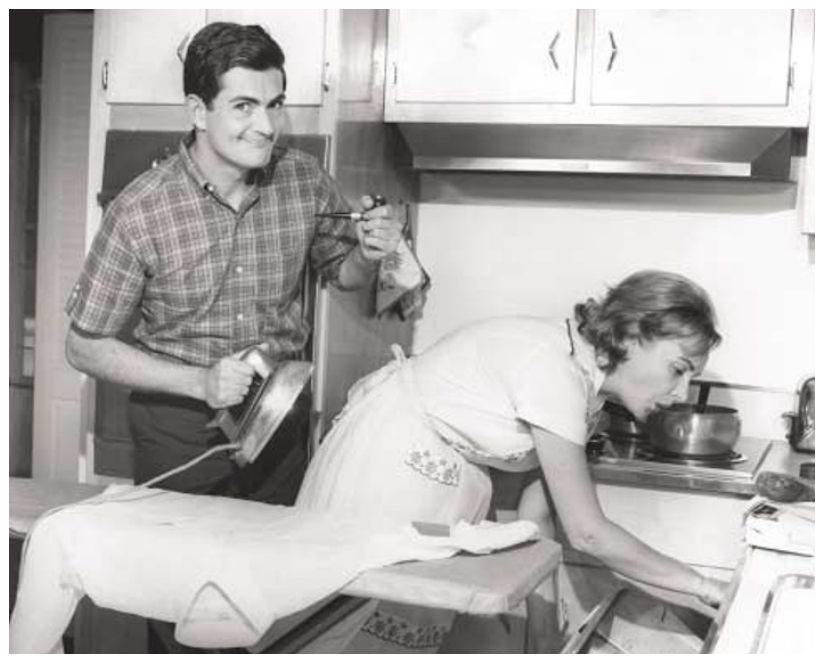

CHEMOTHERAPEUTICS

\section{Opposites don't always attract}

PC-SPES - a herbal preparation that is commonly taken by prostate cancer patients - interferes with cell-cycle arrest and apoptosis, but the precise mechanisms involved have been unclear. But now, Bonham and colleagues, in 6 November issue of Journal of the National Cancer Institute, show that PC-SPES might interfere with microtubule polymerization. The authors show how PC-SPES interacts with microtubule-modulating chemotherapeutic agents, such as paclitaxel, and discuss the implications that these findings have for patients.

To assess the mode of action of PC-SPES, the authors compared the gene expression profile of $\mathrm{LNCaP}$ prostate adenocarcinoma and six other cell lines before and after treatment with PC-SPES. After 48 hours of treatment, 319 transcripts were altered, many of which were involved in either cell cycling, immunity, cell stress or androgen regulation.

However, the expression of one gene - $\alpha$-tubulin, which has a crucial role in cell division - was shown to decrease in all seven cell lines tested. Further analysis by northern blotting showed that $\alpha$-tubulin levels in LNCaP cells decreased by sevenfold after 48 hours, and immunofluorescence showed that the microtubules began to exhibit signs of disruption after just 8 hours of treatment with PC-SPES.

Further investigations revealed that this disruption was directly caused by a reduction in the rate and overall amount of tubulin polymerization. By contrast, $\alpha$-tubulin levels were only marginally reduced in paclitaxel-treated LNCaP cells, microtubules were stabilized, and the rate and overall amount of tubulin polymerization was increased with this treatment. In addition, tumour growth was inhibited to a lesser extent when mice were treated with both PC-SPES and paclitaxel, compared with paclitaxel alone. This indicates that the tubulin dynamics induced by paclitaxel might be antagonistic to those caused by PC-SPES, and that PC-SPES attenuates the effects of paclitaxel.

So, although PC-SPES seems to be a beneficial 'complementary' therapy for prostate cancer patients, when taken in conjunction with conventional chemotherapeutics such as paclitaxel, the efficacy of treatment is reduced. Care should therefore be taken when using complex herbal preparations, about which little is known, in combination with more conventional therapies.

69) References and links Sandra Clark

ORIGINAL RESEARCH PAPER Bonham, M. J et al. Effects of the herbal extract PC-SPES on microtubule dynamics and paclitaxel-mediated prostate tumor growth inhibition. J. Natl Cancer Instit. 94, 1641-1647 (2002) 